\title{
The Effects of Cable Parameters on Over-Voltage at Motor Terminals in the PWM System
}

\author{
YueJun Liu, YanShu Jiang, Xiao Xiao and Hong Xiao \\ School of Automation, Harbin University of Science and Technology, Harbin, China
}

yuejunl2003@126.com

\begin{abstract}
In PWM system, the reflected voltage in the cable transmission process generates overvoltage and damps high-frequency ringing at motor terminals and further stresses the motor insulation, even leads to insulation breakdown in premature period. Firstly, in this paper, the cable equivalent mathematical model was established based on three-phase cable. Then the propagation velocity, the oscillation frequency and the oscillation duration of the pulse in the cable was described by mathematical method, and the effects of cable parameters on over-voltage at motor terminals was analyzed under the conditions of high-frequency. Secondly depending on the simulation by PSpice software, we tested and verified the conclusion by building simulation models.
\end{abstract}

Keywords: PWM system, overvoltage at motor terminals, mathematical model, cable characteristic parameters, reflected voltage

\section{Introduction}

For PWM inverter long cable drive system using switching devices such as IGBT, the PWM pulse-wave voltage brings in voltage reflection phenomenon through the cable transmission, resulting in overvoltage in the motor terminal [1]. The research reveals the extremely uneven voltage points will be brought in motor windings due to the high rate of voltage rise. With the increasing of the cable length between the inverter and the motor, the high-frequency oscillation over voltage is generated in the motor and its amplitude is twice than the inverter voltage. And overvoltage impairs the common-mode voltage, accelerates the motor bearing damage and affects the lifespan of the cable [2,3].

The study mainly analyses the effect of the cable on motor terminal overvoltage. According to the incident wave voltage and reflected wave voltage formula, we predict that the impact of the cable on the motor end of the line voltage is mainly reflected in the motor end of the line voltage amplitude, oscillation frequency and damping time [4]. And the main factor influencing the overvoltage is the length of the cable, and characteristic parameter [5, 6]. Based on constructing a mathematical model of the motor end of the line voltage and the equivalent model of the cable [7], as well as a combination of Pspice, the study analyzes the impact that the cable makes on the motor end of the line voltage.

\section{The Equivalent Model of Cable}

The characteristic parameter of the cable mainly includes inductance per unit length, the capacitance per unit length, the unit length resistance and characteristic impedance. For example, two-wire cable model, shown in Figure 1. $a$ is radius of the cable, $b$ is the distance between the center point of the two lines, is the length of the cable. 


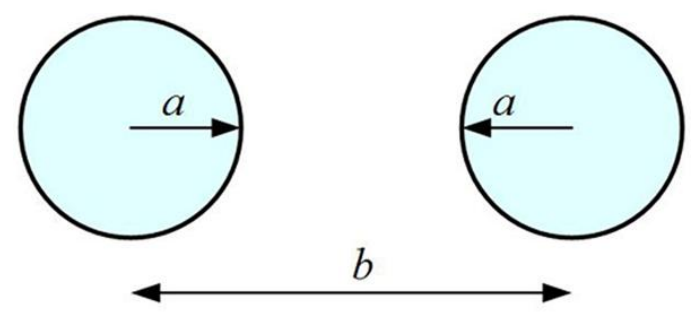

Figure 1. Two-core cable model

The size of the inductance per unit length of the cable is acquired:

$$
L_{C}=\frac{\mu \mu_{0}}{\pi} \ln \left(\frac{b}{2 a}+\sqrt{\left(\frac{b}{2 a}\right)^{2}-1}\right)
$$

$\mu$ is relative magnetic permeability; $\mu_{0}$ is permeability of vacuum

( $\mu_{0}=4 \pi \times 10^{-7} \mathrm{~Wb} / \mathrm{Am}$ ); inductance unite is $H / m$.If $b>>a$, substituting into the vacuum magnetic permeability

$$
L_{C}=0.4 \mu \ln \left(\frac{b}{a}\right)
$$

Which unit is $m H / m$.The capacitance of the cable per unit length is acquired:

$$
C_{C}=\frac{\pi \varepsilon \varepsilon_{0}}{\ln \left(\frac{b}{2 a}+\sqrt{\left(\frac{b}{2 a}\right)^{2}-1}\right)}
$$

$\varepsilon$ is relative permittivity, $\varepsilon_{0}$ is vacuum permittivity $\left(\varepsilon_{0}=8.854 \times 10^{-12} \mathrm{~F} / \mathrm{m}\right)$; the unit of the capacitance is $F / m$.

If $b>>a$, substituting into the vacuum permittivity, $C_{C}$ is acquired:

$$
C_{C}=\frac{28 \varepsilon}{\ln (b / a)}
$$

Which the unit of capacitance is $p F / m$.

The characteristic impedance of the cable is defined as the ratio of the spread of the wave in the positive $x$ direction of voltage and current, for nondestructive cable, it is acquired as the following formulate.

$$
Z_{C}=\sqrt{\frac{L_{C}}{C_{C}}}=120 \sqrt{\frac{\mu}{\varepsilon}} \ln (b / a)
$$

The characteristic impedance of the cable is determined by the geometry of the cable and the insulating characteristics of the medium. And it shows unrelated with the length of the cable, the signal's amplitude, frequency. 


\section{Effect of the Cable Characteristic Parameters on the Voltage}

According to the theory of the transmission line, voltage at any point $\mathrm{x}$ at the cable is acquired $[8,9]$.

$$
\begin{aligned}
v(x, t)= & \frac{Z_{C}}{Z_{S}+Z_{C}} \sum_{k=0}^{\infty}\left\{\left(K_{R} K_{S}\right)^{k} v_{s}\left(t-\frac{x}{v}-2 k \tau\right) \times u\left(t-\frac{x}{v}-2 k \tau\right)\right\}+ \\
& \frac{Z_{C}}{Z_{S}+Z_{C}} \sum_{k=0}^{\infty}\left\{K_{R}^{k+1} K_{S}^{k} v_{s}\left(t+\frac{x}{v}-2(k+1) \tau\right) \times u\left(t+\frac{x}{v}-2(k+1) \tau\right)\right\}
\end{aligned}
$$

The reflection coefficient of the load side is $K_{R}$.

$$
K_{R}=\frac{Z_{R}-Z_{C}}{Z_{R}+Z_{C}}
$$

The reflection coefficient of the output terminal is $K_{S}$.

$$
K_{S}=\frac{Z_{S}-Z_{S}}{Z_{S}+Z_{S}}
$$

Propagation velocity is $v$, and transmission delay is $\tau$.

\subsection{Voltage Amplitude}

The voltage at any point $\mathrm{x}$ at the cable is acquired.

$$
V(x, s)=V^{+}(x, s) e^{-\gamma x}\left[1+K_{R} e^{-2 \gamma(l-x)}\right]
$$

For any load, $\gamma=j \beta$, while $V^{+}$is equal to $V^{+}<0$, based on Euler's formula $e^{j \theta}=\cos \theta+j \sin \theta$, next equation is acquired :

$$
V(x, s)=\hat{V^{+}}(x, s) e^{-j \beta x}\left[1+K_{R} \cos (2 \beta(l-x))-j K_{R} \sin (2 \beta(l-x))\right]
$$

So the voltage amplitude of any point $\mathrm{x}$ on the cable be acquired:

$$
V(x)=V^{+} \sqrt{1+K_{R}^{2}+2 K_{R} \cos [2 \beta(l-x)]}
$$

When the cable is connected to the motor, since the motor impedance is much larger than the cable's, the cable terminal is equal to open. This moment $K_{R}=1$, and the voltage of a point $\mathrm{x}$ on the cable is acquired:

$$
\begin{aligned}
V(x) & =V^{+} \sqrt{2+2 \cos [2 \beta(l-x)]} \\
& =\mid 2 V^{+} \cos [2 \beta(l-x)]
\end{aligned}
$$

In the motor end, $x=l, V(l)=|2 V|^{+}$, theoretically, the maximum value of the over voltage caused by cable is more than twice the voltage of sender. 


\subsection{Propagation Velocity}

According to the analysis, wave transmission speed is a function which includes unit length of the cable inductance $\left(L_{C}\right)$ and per unit length of cable capacitance $\left(C_{C}\right)$. If $\mu=1$ (Cable insulation), substituting $L_{C}$ and $C_{C}$ into the function, the propagation velocity is a function which contains Relative permittivity and speed of light.

$$
v= \pm \frac{1}{\sqrt{L_{C} C_{C}}}=\frac{1}{\sqrt{\mu \mu_{0} \varepsilon \varepsilon_{0}}}=\frac{c}{\sqrt{\mu \varepsilon}}=\frac{c}{\sqrt{\varepsilon}}
$$

$c=\sqrt{\mu_{0} \varepsilon_{0}}=2.998 \times 10^{8} \mathrm{~m} / \mathrm{s}$ is as well as the speed of light. And the unit is $\mathrm{m} / \mathrm{s}$. It can clearly be seen that the speed of pulse is only determined by the relative permittivity of the material of the cable insulation $\varepsilon$. The period of reflection is determined by the wave propagation velocity in the cable. The period is obtained:

$$
\tau=\frac{l_{c}}{v}
$$

And unite is $s$. So the longer the cable, the longer duration that inverter output pulse reaches the motor end, the slower the response speed.

\subsection{Oscillation Frequency}

Figure 2 (The line voltage of the motor end) is a picture that Motor terminal voltage waveform of the curve changes with time. For Figure 2 analyze the Oscillation frequency.

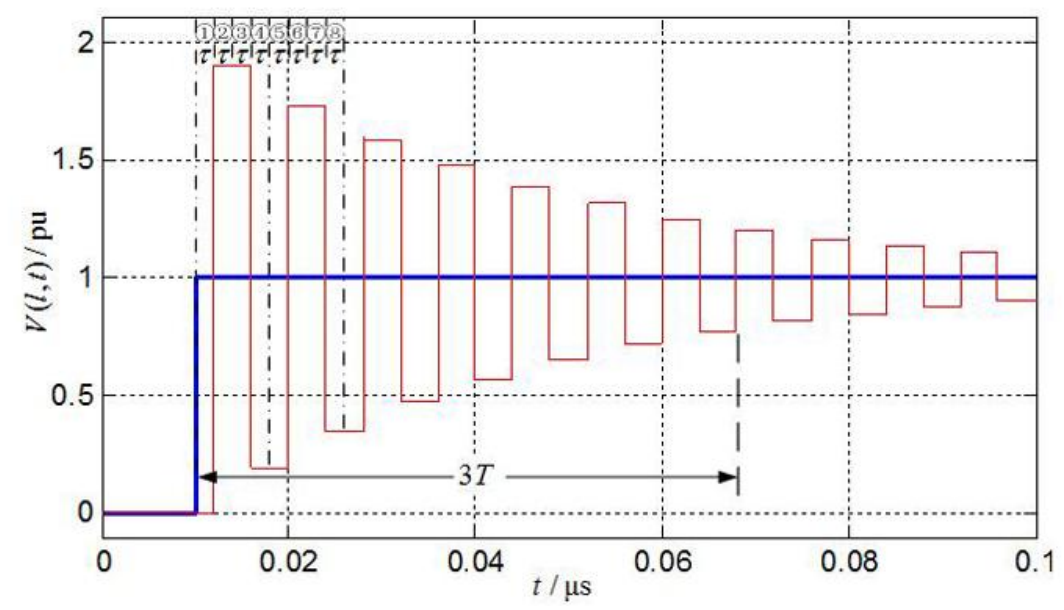

Figure 2. The line voltage of the motor end

In the Figure 2, (1) represents the first period that the inverter output pulse takes to transmit to the motor end. The pulse is called first incident and takes $\tau$ to arrive at the motor end. At this time, parasitic capacitance of the motor is charged.

(2) represent the period that the first reflected wave takes to transmit to the drive end at speed of $v$. This pulse is called the first reflected wave and takes $\tau$ to arrive. The phase of the reflected wave is the same as the incident. At this moment, the voltage of the motor end remains unchanged, as shown in Figure 2 (The line voltage of the motor end). 
(3) represents the period that the inverter reflected voltage takes to transmit from the inverter to the motor end, called the second incident and takes $\tau$ to arrive. If $K_{R}$ is equal to positive one, the minimal line voltage of the motor end called zero voltage is obtained. And this phenomenon is termed total reflex.

(4) represents a time period when the reverse voltage on the motor side takes $\tau$ to come back to the driver. The pulse is called the second reflected wave and forms the third incident wave

In this cycle, the distance of pulse transmission is four times more than the length of the cable $l_{c}$ every $\tau$, the total time $T_{\text {cycle }}$ :

$$
T_{\text {cycle }}=4 \tau=4 l_{c} \sqrt{L_{C} C_{C}}
$$

The Figure 3 shows the correlation of oscillation duration and cable length.

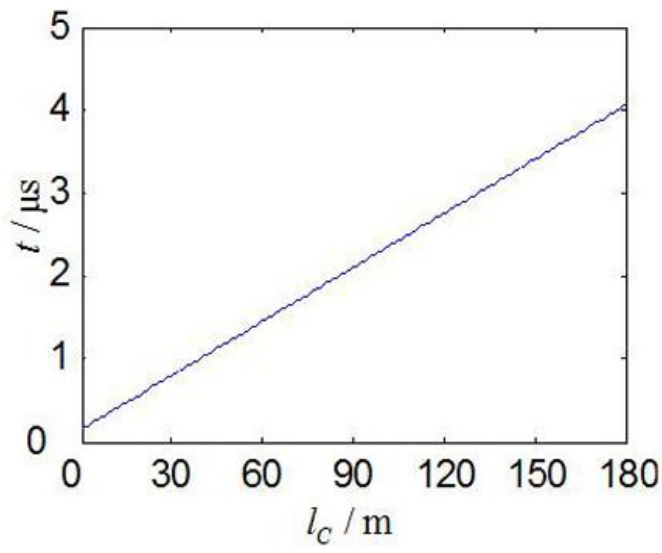

Figure 3. The relationship of the oscillation duration and the cable length

The oscillation frequency is the reciprocal of the oscillation period, shown as formula (39). Evidently, the oscillation frequency is the reciprocal of the length of the cable, shown as Figure 4. The longer the cable, the lower oscillation frequency is; the shorter the cable, the higher oscillation frequency is.

$$
f_{c}=\frac{1}{T_{\text {cycle }}}=\frac{1}{4 l_{c} \sqrt{L_{C} C_{C}}}
$$

The pace is determined by the cable characteristic impedance, and the size of the cable parameters $\left(L_{C}\right.$ and $\left.C_{C}\right)$ is determined by the cable geometry, insulation type and conductor spacing.

Visibly, the oscillation frequency of the line voltage in the motor end is only determined by the cable characteristics and unrelated with inverter characteristics or motor characteristics. 


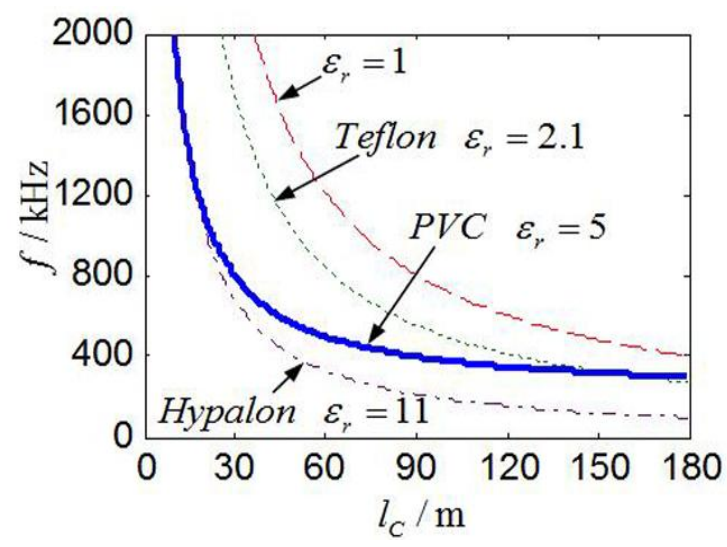

Figure 4. The relationship of the oscillation frequency and the cable length

\subsection{Oscillation Duration}

The greater the resistance, the greater cable damping is; the faster signal attenuation, the shorter oscillation duration is. DC resistance of the unit length can be found in the manual of standard cable. High-frequency oscillation mainly affects cable AC resistance.

The resistance in the lossless line is limited, and usually is neglected. The electric power is stored in the energy storage element (capacitor) in the unit length of lossless line. It is shown as following function:

$$
P=E v=\frac{1}{2}\left(v_{f}\right)^{2} C_{C} \frac{1}{\sqrt{L_{C} C_{C}}}=\frac{\left(v_{f}\right)^{2}}{2 Z_{C}}
$$

Since the cable insulation resistance is much larger than the cable lead resistance and the cable insulation conductance is much smaller than the cable lead conductance, the motor will not consume the power transported by the pulse in the cable and eventually the energy in the energy storage element consumes in the cable resistance.

For the open the terminal, the resistance loss of $d x$ in the cable is obtained:

$$
\frac{2 v_{f} d\left(v_{f}\right)}{2 Z_{C}}=-\left(\frac{v_{f}}{2 Z_{C}}\right)^{2} r_{C} d x
$$

Integrate the both sides of the formula (3-11):

$$
\frac{v}{v_{0}}=e^{-\left(r_{C} x / Z_{C}\right)}
$$

Which $v_{0}$ is the voltage amplitude of the high frequency oscillation caused by the inverter output pulse; $v$ is the voltage amplitude of the cable end; $x$ is the distance that the pulse walks in the cable.

Considering the skin and proximity-effect, the function about the AC resistance of solid copper conductors per lengthen and the cable oscillation frequency is obtained as (3-13):

$$
r_{C}=K_{P} K_{s k i n} r_{d c}=K_{P}\left(\frac{d_{0} \sqrt{f_{C}}}{4(0.0661)}\right)\left(\frac{4 \rho}{\pi d_{0}^{2}}\right)=\frac{8.28 \times 10^{-8} K_{P} \sqrt{f_{C}}}{d_{0}}
$$


$K_{P}$ represents the effect proximity-effect on resistance. Skin effect on resistance coefficient is $K_{\text {skin }}=d_{0} / 4 \lambda=\left(d_{0} \sqrt{f_{C}} / 4(0.0661)\right), \lambda$ represents the skin-effect depth of conductors currents, $d_{0}$ is the diameter of the cable , $\rho=1 / \sigma, \sigma=5.8 \times 10^{7} \mathrm{~S} / \mathrm{m}$ represents electrical conductivity.

The oscillation duration is obtained:

$$
t_{s}=3 T
$$

The distance $x$ traveled by the pulse in the cable is equal to $v t$, the pulse velocity $v=1 / \sqrt{L_{C} C_{C}}$ and $Z_{C}=\sqrt{L_{C} / C_{C}}$ is substituted into (3-12),

$$
\frac{v}{v_{0}}=e^{-r_{C} v t / Z_{C}}=e^{-t / T}
$$

The time constants $(T)$ is obtained:

$$
T=Z_{C} /\left(r_{C} v\right)=L_{C} / r_{C}
$$

Analyzing the formula (3-16), the time constant of the bundling cable is very small. The reflected wave travels from the driver to the motor end, and its attenuation process will be ended swiftly. Likewise, the small diameter cable has big DC resistance, short time constant and relative reflected wave attenuation speed swift.

Thus, the motor terminal line voltage oscillation duration is obtained:

$$
t_{s}=3 T=3 L_{C} / r_{C}=\frac{3 L_{C} d_{0}}{8.28 \times 10^{-8} K_{P} \sqrt{f_{C}}}
$$

Figure 5 illustrates the correlation between the different oscillation frequencies and oscillation duration under the different types of cable by using the formula (3-17). With the oscillation frequency increasing, the faster the motor terminal line voltage attenuate, the shorter the oscillation duration is. The shorter the cable, the higher the oscillation frequency and relatively the faster the attenuation is.

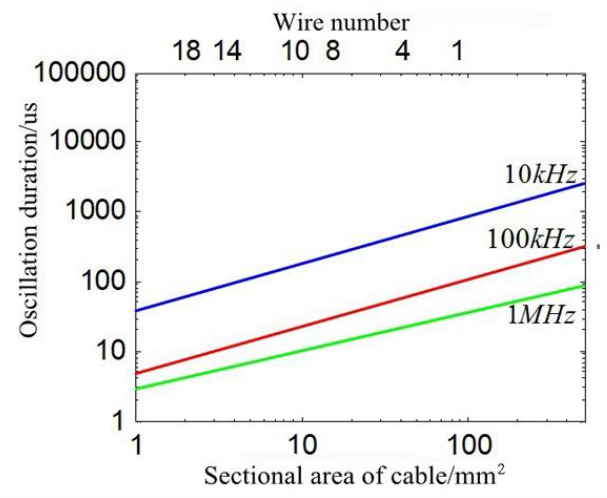

Figure 5. Calculated damping time of reflected wave transient for PVC bundled conductor cables 
At low frequencies, skin effect and proximity effect is not remarkable, and the resistance $r_{C}$ is mainly the DC resistance $r_{d c}$. The larger the DC resistance, the faster reflected wave attenuation is. So the reflected wave decays swiftly in the cable whose diameter is small.

At high frequencies, skin and proximity effect is remarkable, AC resistance is much larger than the DC resistance. So the resistance $r_{C}$ is mainly from AC resistance. AC resistance largely depends on cable oscillation frequency.

With the cable growing, PWM carrier frequency grows, the line voltage oscillation duration prolongs, and the possibility that the overvoltage appear in the motor end increases.

\section{Simulations Analysis in Pspice}

Simulations can be achieved by correcting cable model the transmission time (TD), $\tau=l_{c} / 1.41 \times 10^{8}$. In the following simulation, all the pulse ascent times are $t_{r}=1.6 u s$. The simulation result is shown in Fig.6 (The relationship of the motor terminal voltage and the cable length).
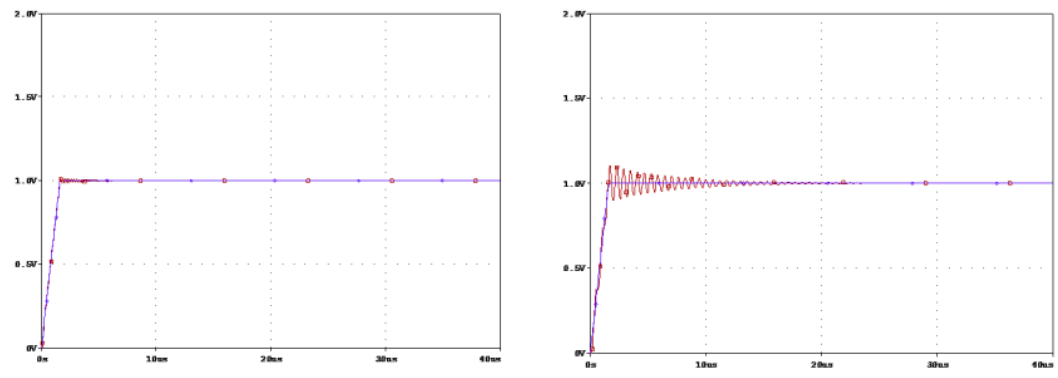

(a) $l_{C}=10 m$

(b) $l_{C}=30 m$
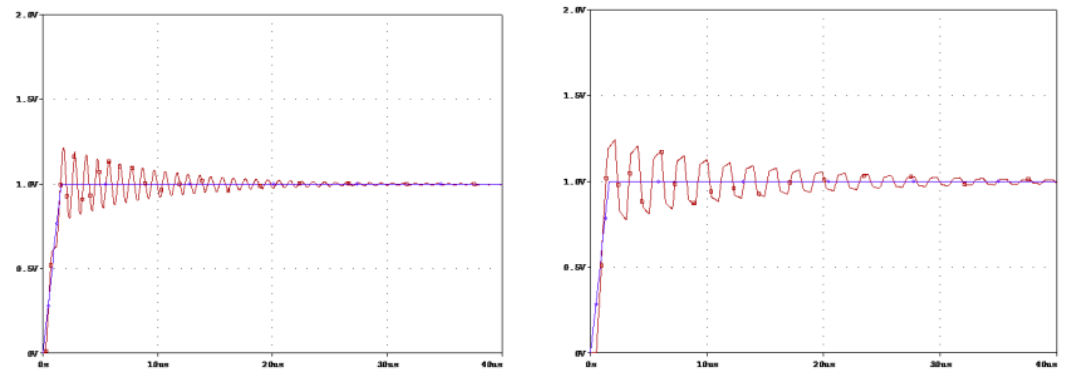

(c) $l_{C}=50 m$

(d) $l_{C}=100 m$

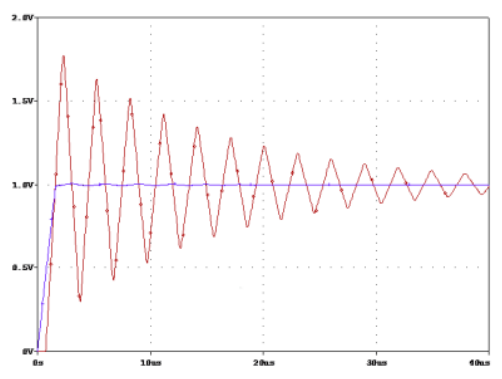

(e) $l_{C}=150 m$

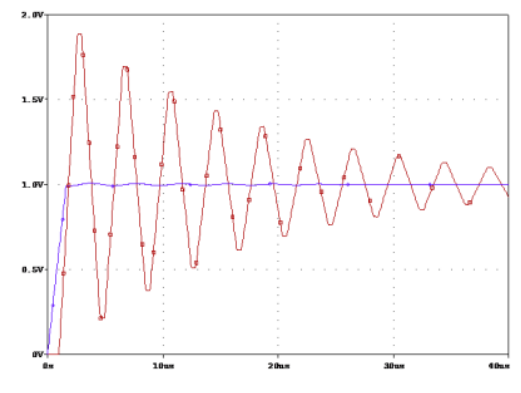

(f) $l_{C}=200 m$

Figure 6. The relationship of the motor terminal voltage and the cable length 


\section{Conclusion}

It is obviously that in the same rise time, with the gradual growing of the cable length, the line voltage of the motor end gradually increases and overvoltage hazard gradually goes up; and the oscillation cycle of the line voltage in the motor end gradually increases and the duration of the oscillation is elongated. If the duration of the inverter output pulse is less than the duration of the line voltage oscillation of the motor end. More than $2 p u$ transient voltage will be generated at the motor end.

\section{References}

[1] T. Weidinger, "Common mode oscillation in electrical drive system", The International Journal for Computation and Mathematic in Electrical and Electronic Engineering, vol. 29, no. 1, (2010), pp. 221-234.

[2] D. -H. Hwang, K. -C. Lee, J. -W. Jeon, Y. -J. Kim, M. -H. Kim and D. -H. Kim, "Analysis the voltage distribution in stator winding of IGBT PWM inverter-fed induction motor", Proceedings of the IEEE International Symposium in Industrial Electronic 2005 (ISIE 2005), vol. 3, (2005) June, pp. 945-950.

[3] J. Diu, K. Liu and Y. Wu, "A pulsed power supply based on power semiconductor switches and transmission line transformer", Dielectrics and Electrical Insulation, IEEE, vol. 14, Issue 4, (2007) August, pp. 927-930.

[4] S. Amarir and K. Al-Hadad, "A modeling technique to analyze the impact of inverter supply voltage and cable length on industrial motor-drives", Power Electronic IEEE, vol. 23, Issue 2, (2008) March, pp. 753762.

[5] G. Mugala, R. Erikson and P. Patterson, "Dependence of XLPE insulated power cable wave propagation characteristics on design parameters", Dielectrics and Electrical Insulation, IEEE, vol. 14, Issue 2, April (2007), pp. 393-399.

[6] R. Prazyan, P. Patterson, H. Edin, R. Erikson and U. Gafyert, "Extraction of high frequency power cable characteristics from S-Parameter measurement", Dielectrics and Electrical Insulation, IEEE, vol. 11, Issue 3, (2004) June, pp. 461-470.

[7] J. W. Palchefsky, "A two-port equivalent model for a transmission line with distributed source", Nuclear Science, IEEE, vol. 34, Issue 6, (1987) December, pp. 1487-1493.

[8] T. S. Pang, P. L. So and A. Kamarul, "Common-model current propagation in power line communication networks using multi-conductor transmission line theory", Power Line Communication and Its Applications, 2007, ISPLC07,IEEE International Symposium, vol. 15, (2007) March, pp. 517-522.

[9] J. E. Jeffery, "Impendence control of conductors acting as transmission in lines in printed board for high frequency digital application", Circuit World, vol. 23, Issue 2, (1997), pp. 22-29. 
International Journal of Control and Automation Vol.7, No.3 (2014) 